\title{
COMPOSITION DEPENDENCE OF THE ENERGY GAP IN HgFeSe - OPTICAL VERIFICATION
}

\author{
W. SzUSzKIEWICZ \\ Institute of Experimental Physics, Warsaw University \\ Hoża 69, 00-681 Warszawa, Poland \\ C. Julien, M. BaLkanski
}

Laboratoire de Physique des Solides, Université Paris VI, France

K. DybKo and B. Witkowska

Institute of Physics, Polish Academy of Sciences, Warszawa, Poland

\begin{abstract}
Infrared reflectivity was investigated for the mixed $\mathrm{Hg}_{1-x} \mathrm{Fe}_{x}$ Se crystals for $x<0.1$. The theoretical analysis of the experimental curves suggests opening of the energy gap for the composition $x=0.087$ at the temperature close to $80 \mathrm{~K}$.
\end{abstract}

PACS numbers: 71.25.Tn, 71.55.-i, 78.30.Fs

The purpose of the present work was to study the infrared optical properties of $\mathrm{Hg}_{1-x} \mathrm{Fe}_{x} \mathrm{Se}$ mixed crystal in order to determine the composition dependence of the energy gap in this material.

It is believed that the absolute value of the negative energy gap for $\mathrm{Hg}_{1-x} \mathrm{Fe}_{x} \mathrm{Se}$ decreases with increasing Fe content and at low temperatures is equal to zero for the mixed crystal composition corresponding to about $15 \%$ of $\mathrm{Fe}$. This value was given in some recent papers analysing the results of the optical measurements performed at temperatures above $100 \mathrm{~K}$ [1] and the helium temperature quantum oscillations observed for the magnetoplasma waves [2].

In this paper we analysed the infrared reflectivity spectra taken with the use of the Bruker FTIR 113v spectrometer. The measurements were performed on different $\mathrm{Hg}_{1-x} \mathrm{Fe}_{x} \mathrm{Se}$ mixed crystals in a wide temperature range $(4.5 \mathrm{~K} \leq$ $T \leq 295 \mathrm{~K}$ ). The crystals were obtained by the Bridgman method in the Institute of Physics, Polish Academy of Sciences in Warsaw, the sample composition $(0 \leq x \leq 0.1)$ was determined by the energy dispersive $\mathrm{X}$-ray fluorescence analysis. The Hall measurements at various temperatures have also been performed for the most of crystals .

The Fe level energy at $4.2 \mathrm{~K}$ increases with the Fe concentration. This effect in the high-doping range $(x<0.01)$ suggested in [2] is two times stronger than that given in [3]. Morever, according to [2] the important nonlinear effect in such 
dependence could be expected for the greater Fe content so this increase should be much slower in the mixed crystal range.

Both suggestions given in [2] were supported by the results of our measurements. The composition dependence of the Fe level energy at $T=4.5 \mathrm{~K}$ was directly observed for $x<0.01$ as a shift of the relevant structures in the reflectivity spectra taken in the vicinity of expected Fermi level position (for the details of the method, see $[4,5])$. For the sample with $x=0.087$ at $T=4.5 \mathrm{~K}$ the Fermi energy value corresponding to measured electron concentration agrees fairly well with the predictions taken from [2].

To reproduce theoretically the reflectivity spectra in the plasma edge region the simplest possible model of the dynamic dielectric function with the classical Drude-Zener free-carrier term was applied.

The temperature dependence of the plasma edge measured for the samples corresponding to the high Fe doping region $(x<0.01)$ is well described by the results of theoretical calculations assuming that the band structure parameter values are the same as those for HgSe. The plasma frequency increases monotonously with increasing temperature, the average value of the relevant coefficient describing the temperature changes is equal to about $5.1 \times 10^{-2} \mathrm{meV} \mathrm{K}^{-1}$. This is the typical situation for the narrow-gap semiconductor with the inverted band structure. For the sample with $x=0.053$ (for which the value of the energy gap should be much smaller, but still negative) the increase of the plasma frequency value is similar to the previous one at temperatures $T \geq 120 \mathrm{~K}$. At low temperatures the mean value of the above-mentioned coefficient is two times smaller. It seems also that its value decreases significantly with decreasing temperature. For the sample with $x=0.087$ a non-monotonous temperature dependence of the plasma frequency value was observed. The plasma energy position decreases significantly from $85.8 \mathrm{meV}$ to $79.4 \mathrm{meV}$ with increasing temperature from $4.5 \mathrm{~K}$ to about 80-100 K. A further temperature increase causes the "normal" plasma frequency behavior (Fig. 1).

The Hall measurements performed for the sample with $x=0.087$ demonstrate an unusual temperature dependence of the electron concentration: the concentration reached the minimal value at about $90 \mathrm{~K}$ and the difference between the concentrations obtained at $4.2 \mathrm{~K}$ and $90 \mathrm{~K}$ was equal to $20 \%$.

We have tried to reproduce this last dependence supposing that due to the high $\mathrm{Fe}$ concentration in the mixed crystal the density of states (DOS) related to $\mathrm{Fe}$ corresponds not to one discrete level, but to a broad band. The Gaussian shape of the DOS was assumed, the peak energy position has been taken from [2]. All band structure parameter values (except the energy gap) were taken the same as those for $\mathrm{HgSe}$. The electron concentration at $T=4.2 \mathrm{~K}$ and the total $\mathrm{Fe}$ concentration (directly measured in our experiments) were fixed. The supplementary condition was that according to the experimental results the electron concentration at $T=$ $295 \mathrm{~K}$ should be higher than that at $4.2 \mathrm{~K}$. The full width at half maximum (FWHM) of the DOS function and the energy gap were used as fitting parameters. 


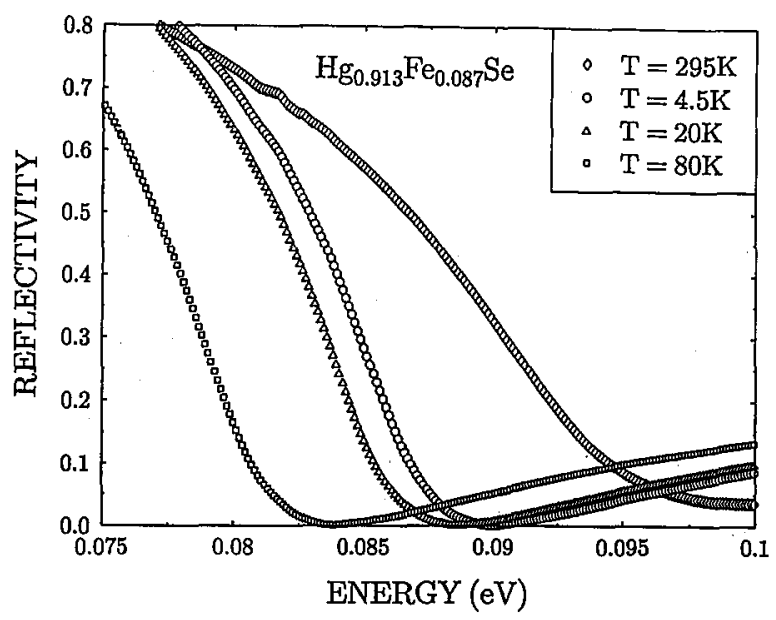

Fig. 1. The temperature dependence of the plasma edge in $\mathrm{Hg}_{1-x} \mathrm{Fe}_{x} \mathrm{Se}$ observed for the sample with $x=0.087$ (the composition close to the one corresponding to the exact zero value of the energy gap at $T=4.5 \mathrm{~K}$ in this material).

The following conclusions result from the model calculations described above:

1. Minimum in the concentration temperature dependence can be reproduced only for a relatively narrow energy gap value and FWHM value ranges.

2. FWHM of Fe DOS function could not be smaller than about $10 \mathrm{meV}$ and greater than about $30 \mathrm{meV}$.

3. Energy gap value at $T=4.2 \mathrm{~K}$ should be small and negative $\left(-70 \mathrm{meV} \leq E_{\mathrm{g}} \leq-0.30 \mathrm{meV}\right)$.

4. The best fit values are FWHM $=25 \mathrm{meV}$ and $E_{\mathrm{g}}=-50 \mathrm{meV}$ at $T=4.2 \mathrm{~K}$.

Within the frames of the present model it was not possible to reproduce the electron concentration decrease with increasing temperature greater than $10 \%$ (the experimental result is 20\%). It seems that the DOS function for Fe could have more complicated form than that assumed for the numerical estimations.

Our final conclusion is that the band structure of $\mathrm{Hg}_{1-x} \mathrm{Fe}_{x}$ Se changes more rapidly with increasing $\mathrm{Fe}$ content than it was believed till now. We estimate that at the helium temperature the "crossing point" corresponds to about $10 \%$ of $\mathrm{Fe}$ in the mixed crystal. In our opinion the first experimental evidence of the broadening of the $\mathrm{Fe}$ "level" enabling the quantitative estimations of this effect was also found.

\section{Acknowledgments}

We would like to express our gratitude to Prof. A. Mycielski for his interest in this work and his valuable remarks and to Dr. W.D. Dobrowolski for his help in the crystal composition analysis. One of us (W.Sz.) would like to thank Laboratoire de Physique des Solides de l'Université Pierre et Marie Curie for the financial support during his stay in Paris. 
760 W. Szuszkiewicz, C. Julien, M. Balkanski, K. Dybko, B. Witkowska

\section{References}

[1] S.Yu. Paranchich, L.D. Paranchich, V.N. Makogonenko, S.V. Lototskaya, Fiz. Tekh. Poluprovodn. 24, 225 (1990).

[2] V.D. Prozorovskij, I.Yu. Reshidova, S.Yu. Paranchich, L.D. Paranchich, Fiz. Tverd. Tela 32, 3290 (1990).

[3] F.S. Pool, J. Kossut, U. Debska, R. Reifenberger, Phys. Rev. B 35, 3900 (1987).

[4] W. Szuszkiewicz, G. Karczewski, M. Jezewski, B. Witkowska, A. Mycielski, in: The Physics of Semiconductors, Proc. 20th Intern. Conf. on the Physics of Semiconductors, Thessaloniki 1990, Ed. E.M. Anastassakis, J.D. Joannopoulos, World Scientific, Singapore 1990, p. 1867.

[5] W. Szuszkiewicz, C. Julien, M. Balkanski, M. Arciszewska, B. Witkowska, A. Mycielski, Acta Phys. Pol. A 80, 437 (1991). 